\title{
Frustrated self-assembly of dendron and dendrimer-based supramolecular liquid crystals $\dagger$
}

\author{
Raffaele Mezzenga, ${ }^{* a b}$ Janne Ruokolainen, ${ }^{c}$ Nadia Canilho, ${ }^{a}$ Edis Kasëmi, ${ }^{d}$ Dieter A. Schlüter, ${ }^{d}$ Won Bo Lee ${ }^{e}$ \\ and Glenn H. Fredrickson ${ }^{e}$
}

\begin{abstract}
A new "inverted" topological configuration is demonstrated both experimentally and theoretically for self-assembled dendron and dendrimer-based supramolecular liquid crystals in which the dendrons/ dendrimers occupy the continuous domain and the ionically attached pendant chains are confined in discrete domains. All previous studies on dendrimer and dendron-based liquid crystals have reported "normal" liquid crystalline configurations in which the dendritic templates occupy discrete domains (in spherical or columnar phases) or continuous struts (in bicontinuous cubic phases), while the pendant chains occupy the continuous space-filling domain. These surprising results mandate a re-examination of the packing mechanisms for this important class of materials and open new routes to unique nanostructures of possible use in existing and emerging technologies.
\end{abstract}

Supramolecular self-assembly of polymers offers nearly limitless possibilities when the architecture of the macromolecular template, the nature of the supramolecular interactions and the diversity of the ligands (tectons) attached to the polymer backbone are fully exploited. ${ }^{1-5}$

Dendrons and dendrimers differ from more traditional macromolecular templates by the fact that their typical size, number of peripheral functional groups which can participate in supramolecular interactions and other physical properties can be systematically tuned by varying their generation. ${ }^{6}$ Another distinguishing property of these branched templates is their intrinsic curvature at the dendritic surface, which is "concave" with respect to the focal point. In previous studies of liquid crystals produced by covalent or supramolecular attachment of pendant chains on dendron or dendrimer surfaces, it has been assumed that the concave dendrimer/pendant chain interface is mirrored in the bulk self-assembled structures. ${ }^{7-23}$ Indeed, the current understanding and molecular models are based on X-ray (SAXS) and electron scattering data, which only provide the reciprocal

${ }^{a}$ Department of Physics and Fribourg Center for Nanomaterials, Ch. du Musée 3, CH-1700 Fribourg, Switzerland.E-mail: raffaele.mezzenga@, unifr.ch

${ }^{b}$ Nestlé Research Center, Vers-Chez-les-Blancs, 1000, Lausanne 26 Switzerland

${ }^{c}$ Department of Engineering Physics, Helsinki University of Technology, Espoo, Finland

${ }^{d}$ Swiss Federal Institute of Technology, Department of Materials, Institute of Polymers, ETH-Zurich, HCI J 541, CH-8093, Zurich, Switzerland ${ }^{e}$ Materials Research Laboratory, University of California, Santa Barbara, CA, 93106, USA space signature of the crystalline space group without allowing the direct identification of domains ("Babinet" Principle). ${ }^{24}$

The accepted self-assembly scheme of dendritic liquid crystals, which postulates interfaces concave towards the focal points of dendrons and dendrimers, may be "frustrated" by a reduction in the volume fractions of the pendant chains. This would favour bending the interfaces in the opposite direction, conferring an "inverted" configuration to the liquid crystalline polymers. To this end, Tschierske et al., have shown that in conventional pendant chain liquid crystalline polymers, the volume fraction of the pendant chains plays an important role in defining the final curvature of the domain interfaces. ${ }^{25,26}$ Furthermore, we have recently demonstrated that in columnar liquid crystalline phases based on dendronized polymer/alkyl tail ionic complexes, an "inverted" configuration (that is pendant chains in the columns), is possible also at large volume fractions of pendant chains. ${ }^{27}$

In order to understand whether this "inverted" self-assembly mechanism is general and also applies to dendrons and dendrimers liquid crystal systems, in which a high intrinsic topological curvature is present, we have synthesized 1st and 2nd generation dendrons and dendrimers carrying terminal ammonium groups and studied the self-assembly of their ionic supramolecular complexes with sulfate alkyl tails by means of reciprocal space techniques (SAXS), direct space techniques (transmission electron microscopy, TEM) and self-consistent field theory simulations (SCFT).

Fig. 1 provides a scheme for the dendritic structures and surfactants investigated, while Table 1 gives the volume fractions of the alkyl tails in the resulting supramolecular complexes (refer to ESI, for details $\dagger$ ).

All the complexes from the DG1-Cx series proved to be lamellar due to the crystalline nature of DG1 (see ESI $\dagger$ ). More interestingly, the complexes from the DG2-Cx series showed the presence of both columnar and lamellar phases, depending on the length of the Cx alkyl tail used. Fig. 2A shows the SAXS spectra recorded for the DG2-Cx series. For the C8 complexes, 


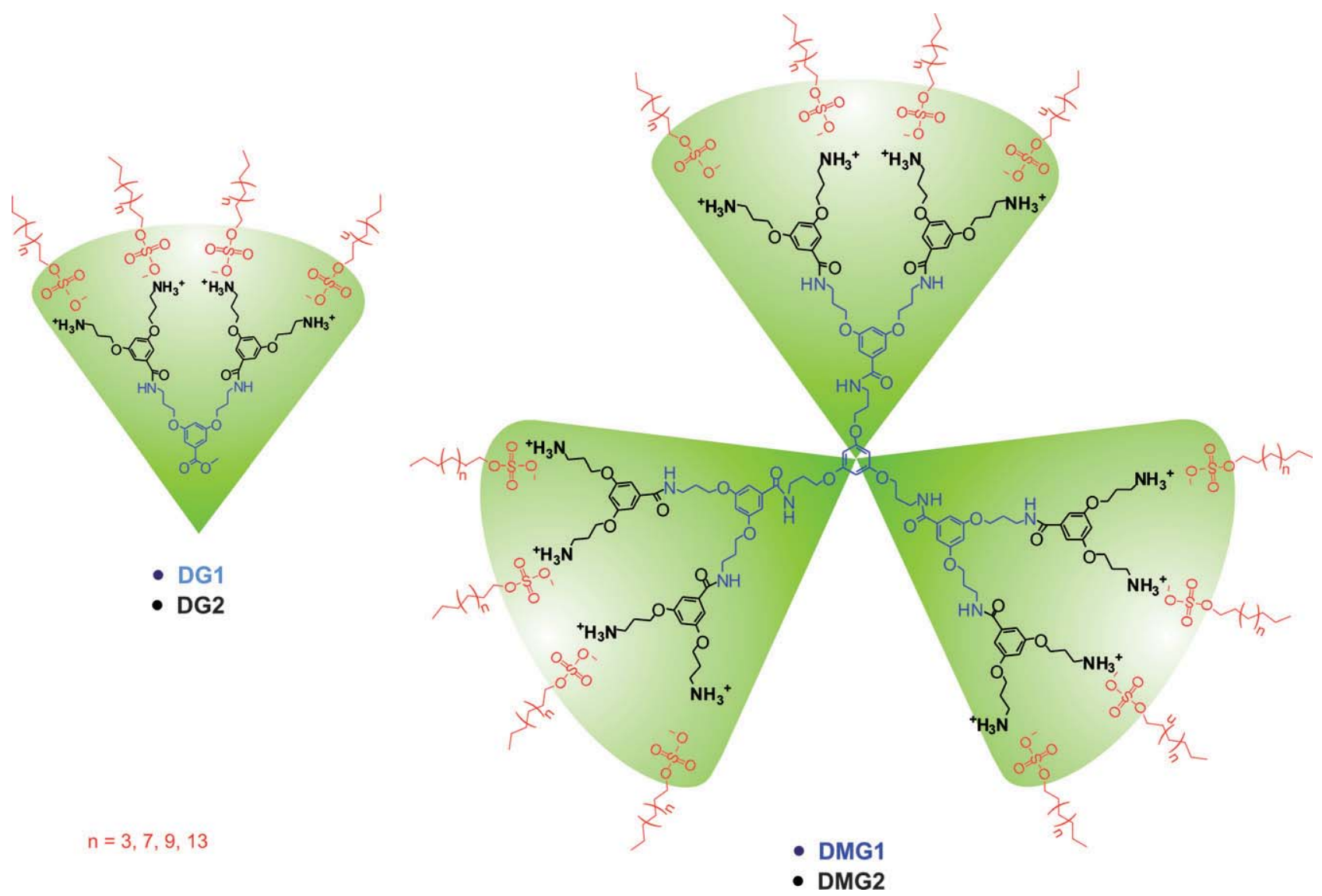

Fig. 1 Schematic drawings of the surfactants, dendrons and dendrimers used in the present study. First and second generation dendrons are identified as DG1 and DG2, respectively; first and second generation dendrimers as DMG1 and DMG2, respectively. Second generation dendrons and dendrimers are entirely drawn; the corresponding first generations, (excluding ammonium charges) are highlighted in blue. The interface between the apolar alkyl tails and polar dendrons/dendrimers is drawn beyond the sulfate polar head. Cx identifies the sulfate alkyl tails with $8,12,14$, and 18 carbons respectively.

Table 1 Alkyl tail volume fractions of the dendron and dendrimer-based complexes $^{a}$

\begin{tabular}{llll}
\hline & DG2 & DMG1 & DMG2 \\
\hline C8 & 0.36 & 0.36 & 0.32 \\
C12 & 0.43 & 0.45 & 0.4 \\
C14 & 0.47 & 0.48 & 0.43 \\
C18 & 0.53 & 0.54 & 0.49
\end{tabular}

${ }^{a}$ Respective densities in $\left(\mathrm{g} / \mathrm{cm}^{3}\right)$ measured by a helium pycnometer $\mathrm{d}_{\mathrm{C} 8}=$ $1.05 \mathrm{~d}_{\mathrm{C} 12}=1.09 \mathrm{~d}_{\mathrm{C} 14}=1.12 \mathrm{~d}_{\mathrm{C} 18}=1.16 \mathrm{~d}_{\mathrm{DG} 2}=1.52 \mathrm{~d}_{\mathrm{DMG} 1}=1.45$ $\mathrm{d}_{\mathrm{DMG} 2}=1.35$.

two broad peaks spaced as $\mathrm{q}_{1}: \mathrm{q}_{2}=1: 1.52$ appear in the corresponding SAXS profile, which are consistent with a poorly ordered rectangular columnar phase with lattice parameters $(a, b)$ $=2.8,3.8 \mathrm{~nm}$. Both complexes obtained with $\mathrm{C} 12$ and $\mathrm{C} 14$ show the typical profile of a columnar hexagonal phase with three reflections spaced as $\mathrm{q}_{1}: \mathrm{q}_{2}: \mathrm{q}_{3}=1: \sqrt{ } 3: 2$, and lattice periods of 3.6 and $4.5 \mathrm{~nm}$, respectively. Finally, the complex obtained with C18 exhibits 4 reflections spaced as $\mathrm{q}_{1}: \mathrm{q}_{2}: \mathrm{q}_{3}: \mathrm{q}_{4}=1: 2: 3: 4$, which is consistent with a well-ordered lamellar phase with period $4.6 \mathrm{~nm}$.

As anticipated, the SAXS data alone are insufficient to assign the discrete domains of the columnar phases to either the alkyl tails or the dendritic templates.
Fig. 2B shows TEM micrographs of the same DG2-Cx series reported in Fig. 2A. Consistent with the SAXS spectra, poorly ordered columnar phases (DG2-C8), ordered columnar hexagonal phases (DG2-C12 and DG2-C14) and lamellar phases (DG2-C18) were found. $\mathrm{RuO}_{4}$ was used as a selective staining agent to mark the aromatic rings present in the dendritic structures, leaving the fully saturated alkyl tails unstained; thus, the clear domains in Fig. 2B are the alkyl tails, while the dark domains identify the dendrons. Very unexpectedly, the alkyl tails are found to form cylinders while the dendrons constitute the continuous domains. The apparent volume fractions in Fig. 2B are not representative of the real volume fractions (Table 1) since the images were taken slightly out of focus to enhance contrast. The characteristic periods in Fig. 2B, however, perfectly match those obtained by SAXS. By systematically varying the focal plane on the TEM, contrast inversion (zebra effect) could be ruled out, confirming the presence of white cylinders throughout the whole of each sample. Furthermore, in order to exclude possible diffusion of $\mathrm{RuO}_{4}$ within the alkyl tail domains and thus confirm chemical staining of the aromatic rings, TEM was repeatedly performed on stained sections over a period of one year from initial staining. Sections were shown to have unaltered contrast, unambiguously confirming the chemical staining of the dendrons and, thus, the presence of alkyl tails in the cylinders. 


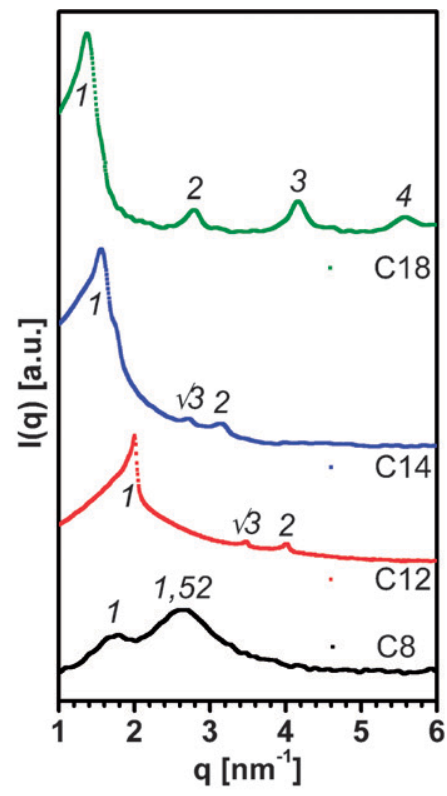

(a)

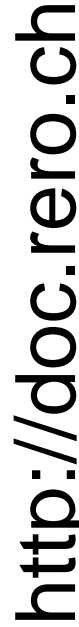

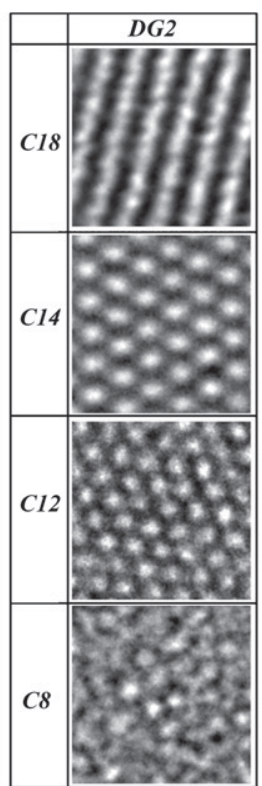

(b)

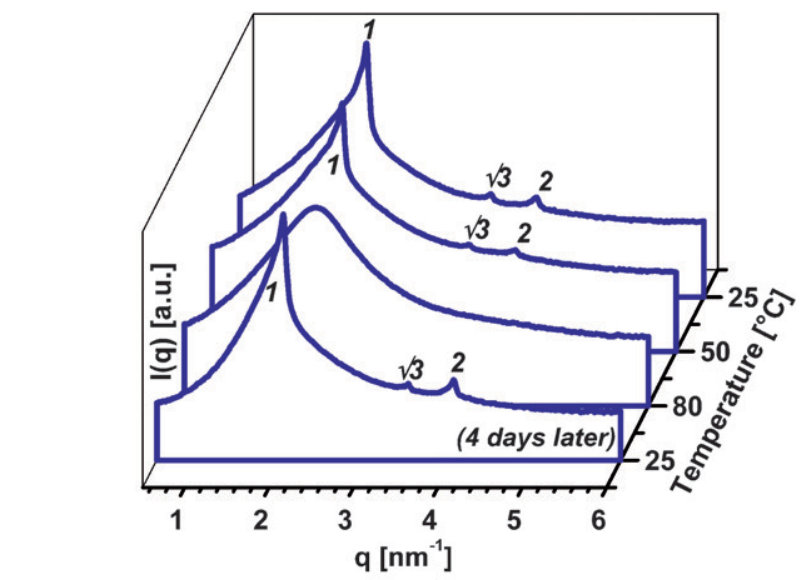

(c)

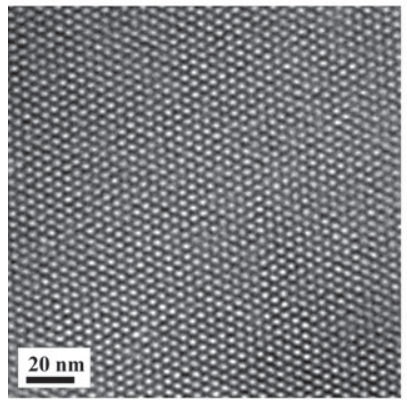

(d)

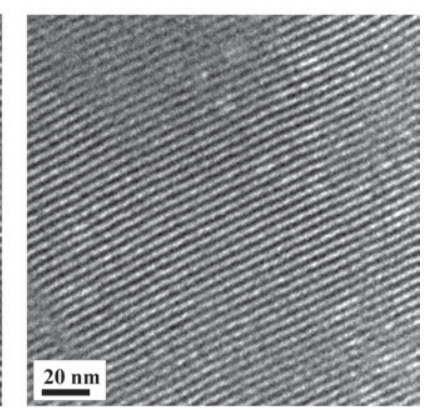

(e)
Fig. 2 (A) SAXS diffractograms for DG2-C8, DG2-C12, DG2-C14, DG2-C18 complexes. (B) High magnification TEM micrographs for DG2-C8, DG2-C12, DG2-C14 and DG2-C18. Each individual image has $25 \times 25 \mathrm{~nm}^{2}$ edges. The white domains represent the alkyl tails while the dark domains are the $\mathrm{RuO}_{4}$-stained dendrons. (C) Temperature-dependent SAXS diffractogram for the DG2-C12 complex. At $25^{\circ} \mathrm{C}$ and $50^{\circ} \mathrm{C}$ a typical columnar hexagonal diffraction pattern is observed. The liquid crystalline structure is cleared at the order-disorder transition temperature $\left(80{ }^{\circ} \mathrm{C}\right)$, but is completely recovered after four days at $25^{\circ} \mathrm{C}$,
Amazingly, the structures observed by TEM were found to be identical in annealed and non-annealed samples, indicating thermodynamically stable structures. This was also confirmed by SAXS, in which non-annealed samples and samples in which the liquid crystalline structure was first cleared by temperature and then recovered by annealing, showed exactly the same scattering profile (Fig. 2C). Finally, the most highly ordered structure (DG2C14) showed remarkable long-range order spanning $\sim \mu \mathrm{m}$ length scales, which represent three orders of magnitude more than the corresponding hexagonal lattice period (see Fig. 2D and 2E).

To verify whether these findings also apply to dendrimers, TEM and SAXS measurements were also conducted on the first and second generation dendrimer series DMG1-Cx and DMG2-Cx.

Fig. 3A shows the micrographs obtained for the various dendrimer/sulfate alkyl tail complexes. Again, white alkyl tail based hexagonally packed cylinders were observed for the DMG1-C12, DMG1-C14, DMG2-C12 and DMG2-C14 complexes. At larger volume fractions of alkyl tails (C18), similarly to the dendron case, lamellar phases were observed, with periods of 4.7 and $5.0 \mathrm{~nm}$ for DMG1-C18 and DMG2-C18, respectively. While ordering was poor for some of the columnar hexagonal phases, good long-range order was observed in the case of DMG1-C12. Fig. 3B shows a typical defect-rich region of the DMG1C12 complex arising at the boundary of two low angle tilted grains $\left(\sim 10^{\circ}\right)$, while Fig. $3 \mathrm{C}$ shows the longitudinal projection of the aligned cylinders. Interestingly, unlike the dendron case, the C8 complexes resulted in homogenous isotropic systems as confirmed by SAXS (see ESI $\dagger$ ).

Finally, as a general remark for both dendron and dendrimer cases, we note that when approaching the "inverted columnar" $\rightarrow$ "lamellar" order-to-order transition upon increase of the pendant chain volume fraction, evolution in the lattice parameter becomes greatly non-linear with the pendant alkyl tails' contour length. This is reflected by the systematic increase in the columnar lattice spacing observed for all the complex series when going from $\mathrm{C} 12$ to $\mathrm{C} 14$. This increase reaches $0.9 \mathrm{~nm}$ for variations among highly ordered columnar hexagonal phases (see for example DG2-C12 $\rightarrow$ DG2-C14 in Fig. 2A), but can be even larger when substantial lattice distortions occur, such as in the DMG2-C12 $\rightarrow$ DMG2-C14 case (see ESI for systematic variations in lattice parameters as measured by SAXS $\dagger$ ). This large increase in lattice parameter upon $\mathrm{C} 12 \rightarrow \mathrm{C} 14$ increment has been previously reported for dendronized polymers-alkyl tail supramolecular complexes. ${ }^{28}$

The presence of thermodynamically stable structures in dendron and dendrimer-based liquid crystalline systems can be theoretically determined by constructing a suitable chain and interaction model for these systems and searching for low free energy structures by SCFT simulations.

Fig. 4 gives schematics of the macromolecular models used for the SCFT simulations of the various dendron and dendrimer complex series together with the corresponding density profiles at fixed volume fractions of the alkyl tails.

indicating thermodynamically stable liquid crystalline structures. (D) Orthogonal TEM projection for long-range ordered hexagonally packed cylinders in the DG2-C14 complex. (E) Longitudinal TEM projection for hexagonally packed cylinders in the DG2-C14 complex. 


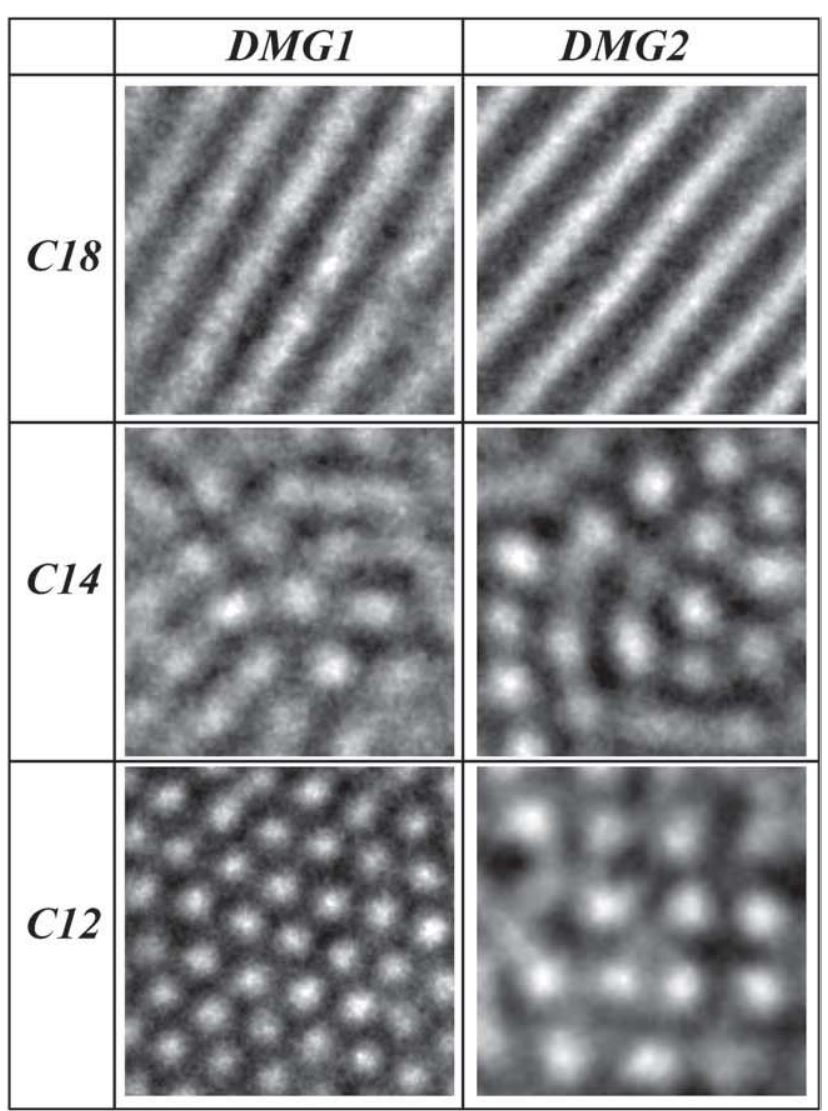

(a)

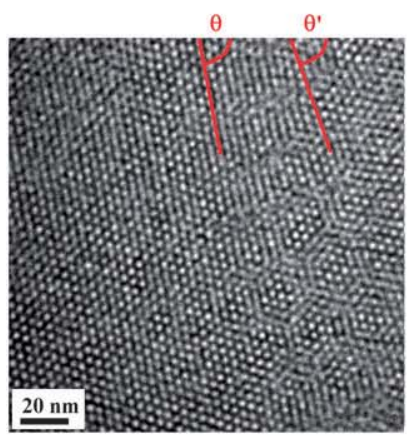

(b)

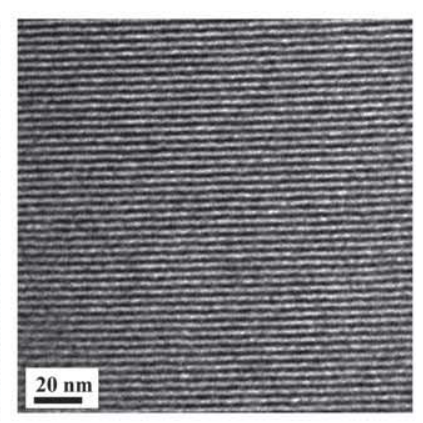

(c)
Fig. 3 (A) High magnification TEM micrographs for DMG1-C12, DMG1-C14, DMG1-C18 and DMG2-C12, DMG2-C14, DMG2-C18 series. Each individual image has $25 \times 25 \mathrm{~nm}^{2}$ edges. The white domains represent the alkyl tails while the dark domains are the $\mathrm{RuO}_{4}$-stained dendrimers. (B) Orthogonal TEM projection for hexagonally packed cylinders in the DMG1-C12 complex. The presence of hexagon-like line defects on the right side of the image arises from the merging of two grains with slightly different tilt angles $\left(\theta-\theta^{\prime} \approx 10^{\circ}\right)$. (C) Longitudinal TEM projection for hexagonally packed cylinders in the DMG1-C12 complex.

Remarkably, disorder $\rightarrow$ hex $\rightarrow$ lam transitions are found for the DMG1 and DMG2 series upon increasing the volume fraction of the alkyl tails. For the DG2 case, the hex $\rightarrow$ lam transition is correctly predicted by SCFT, although the rectangular phase at low volume fraction is predicted to exist only as a metastable structure (not shown). This may indicate that the DG2-C8 rectangular columnar phase is a metastable phase; nonetheless at low alkyl tail fraction, the increased tendency to form columnar phases in dendrons rather than dendrimer-based complexes is confirmed both experimentally and theoretically. This may be a consequence of greater topological restrictions for dendrimers in inverting the dendritic-pendant chain interfaces. The theoretical under-prediction of the volume fractions of the alkyl tails at the order-disorder and order-order phase boundaries may be due to rigidity effects not captured in the Gaussian chain model, or to the neglect of thermal fluctuations inherent in SCFT. ${ }^{29}$

Most importantly, the theory predicts that the columnar structures at low alkyl tail fractions adopt inverted configurations with the alkyl tails in the cylinders, exactly as observed experimentally. This configuration is in conflict with the natural curvature imposed by the dendron/dedrimer architecture, but is favoured by an imbalance of conformational entropy between short alkyl tails and longer dendron segments. Finally, at high enough volume fractions of the alkyl tails $(\approx 0.7)$, "normal" hexagonally packed cylinders (with alkyl tails forming the continuous domain) are predicted for all dendrimer and dendron series, as would be expected when the intrinsic curvature of the dendrimers/dendrons is supported by the conformational entropy gain of long alkyl chains. This physical case has been already imaged by TEM in tapered dendron systems with long pendant chains. ${ }^{30}$ In our present case, experimental evidence of "normal" $\rightarrow$ "inverted" transition was found when increasing the volume fraction of the alkyl tails to 0.7 by using a sulfate butyl oleate double-tailed surfactant (see ESI for details $\dagger$ ).

Our findings mandate a reassessment of postulated selfassembly mechanisms in dendron and dendrimer-based liquid crystals. In order to confine pendant chains into cylinders, dendrons and especially dendrimers must fold back and assume an "octopus"-like configuration, with inverted curvature (see ESI for a sketch $\dagger$ ). This is far from the commonly accepted view of dendritic liquid crystals as "core-shell" particles. In the realm of applications, these novel structures may be used as structurally ideal templates for functional materials. For example, by exploiting the reversible supramolecular nature of the complexes to remove the pendant chains, these systems could serve as templates for ultra-nanoporous materials, in which the density of the pores and their lattice size and organization can be controlled to levels well beyond current alternatives. ${ }^{31}$

\section{Materials and methods}

\section{Synthesis and complex preparation}

The dendrons DG1 and DG2 were synthesized according to known procedures. ${ }^{32}$ The corresponding dendrimers DMG1 and DMG2 were obtained by attaching these dendrons through active ester chemistry to a trifunctional amine core which had been obtained from 1,3,5-trihydroxybenzene and Boc-protected 1-chloro-3-propylamine. All compounds were fully characterized and analytically pure (see ESI for details $\dagger$ ).

Stoichiometric ionic complexes were prepared by adding dropwise to the respective dendritic solutions the equivalent molar mass of monoalkyl sulfate aqueous solutions. The precipitates were dried and annealed under high vacuum at temperatures comprised between the melting temperatures of the 

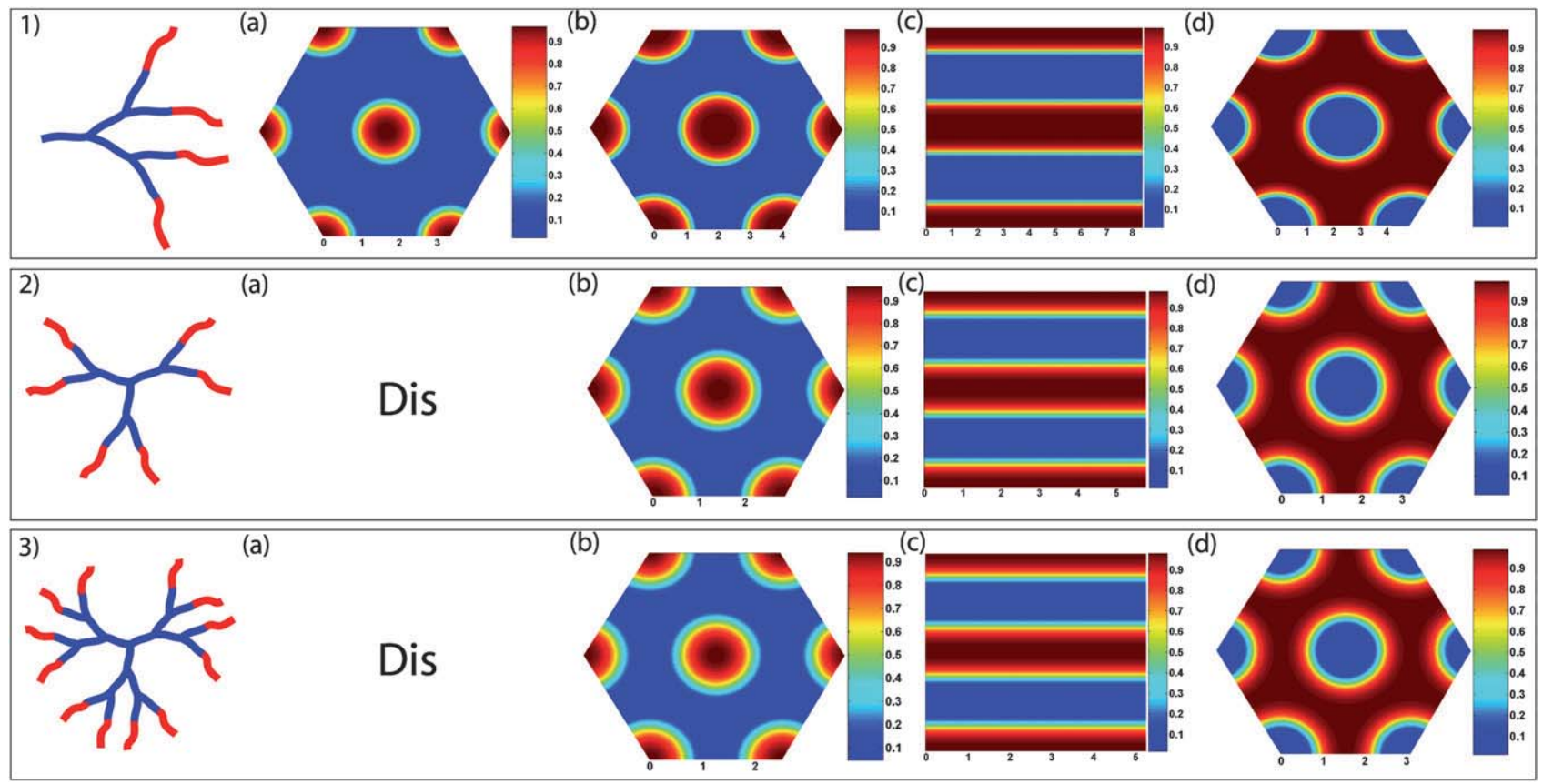

Fig. 4 Segment density profiles calculated by SCFT simulations for dendron and dendrimer-based liquid crystals. Lines 1, 2 and 3 identify the DG2, DMG1 and DMG2 series, respectively. For each series, (a), (b), (c), (d) correspond to a volume fraction of alkyl tails of $0.2,0.3,0.5$ and 0.7 , respectively. The red and blue colours in the density profiles identify the alkyl tails and dendrons/dendrimers, respectively. The scale values in each lattice axis are in the units of the ideal radius of gyration $\left(R_{g}=b \sqrt{N / 6}\right)$ for a linear chain that has the longest end-to-end distance in the corresponding molecule. At very low alkyl tail volume fractions (0.2), the "inverted" columnar hexagonal phase is predicted for DG2, while disordered phases are predicted for the dendrimer series. At higher volume fractions (0.3), "inverted" columnar hexagonal phases are predicted for all the complexes. Lamellar phases are predicted at symmetric volume fractions (0.5), while the "normal" columnar phase is predicted to be stable when the alkyl tails become the majority component (0.7).

alkyl tails and the temperatures of the ordered-disorder transition of the respective liquid crystalline phases $\left(50-80^{\circ} \mathrm{C}\right)$.

\section{Physical characterization}

TEM. Bulk samples were sectioned at $-60{ }^{\circ} \mathrm{C}$ using a Leica Ultracut UCT ultramicrotome and a 25 degree Diatome diamond knife. Sections with thicknesses of $c a$. 50-70 nm were collected on lacey carbon support film grids. In order to provide electron contrast, sections were stained for 2-15 minutes in the vapours of an aqueous solution of $\mathrm{RuO}_{4}$. Bright-field TEM was performed on a FEI Tecnai 12 transmission electron microscope operating at an accelerating voltage of $120 \mathrm{kV}$.

SAXS. Simultaneous small and wide angle X-rays scattering experiments were performed using a SAXSess instrument with a line collimation setup and a $\mathrm{Cu} K \alpha$ radiatin source $(\lambda=$ $0.1542 \mathrm{~nm}$ ). Backgrounds from mica foils used to clamp the samples were subtracted from the diffractograms.

\section{SCFT simulations}

SCFT calculations were performed for the structures shown in Fig. 4 (see ESI for detailst). The molecules are described as branched Gaussian chains with dissimilar segments interacting locally via Flory type interactions $(\chi)$ and an incompressibility constraint. We fixed $\chi=0.05$ and the end-to-end chain length, $\mathrm{N}=1000$, to establish appropriate length and interaction energy scales for different molecules. The SCFT equations were solved numerically in unit cells using advanced algorithms. ${ }^{29}$

\section{Acknowledgements}

Financial support from the Swiss National Science Foundation (SNF) is gratefully acknowledged.

\section{References}

1 J. M. Lehn, Science, 2002, 295, 2400-2403.

2 T. Kato, N. Mizoshita and K. Kishimoto, Angew. Chem., Int. Ed., 2006, 45, 38-68.

3 T. Kato, Science, 2002, 295, 2414-2418.

4 M. R. Hammond and R. Mezzenga, Soft Matter, 2008, 4, 952-961.

5 O. Ikkala and G. ten. Brinke, Science, 2002, 295, 2407-2409.

6 J. M. J. Fréchet, Science, 1994, 263, 1710-1715.

7 V. Percec, C. H. Ahn, G. Ungar, D. J. D. Yeardley, M. Moller and S. S. Sheiko, Nature, 1998, 391, 161-164.

8 V. Percec, W. D. Cho, M. Moller, S. A. Prokhorova, G. Ungar and D. J. P. Yeardley, J. Am. Chem. Soc., 2000, 122, 4249-4250.

9 X. B. Zeng, G. Ungar, Y. S. Liu, V. Percec, S. E. Dulcey and J. K. Hobbs, Nature, 2004, 428, 157-160.

10 R. Martín-Rapún, M. Marcos, A. Omenat, J. Barbera, P. Romero and J. L. Serrano, J. Am. Chem. Soc., 2005, 127, 7397-7403.

11 J. Barberá, B. Donnio, L. Gehringer, D. Guillon, M. Marcos, A. Omenat and J. L. Serrano, J. Mater. Chem., 2005, 15, 4093-4105.

12 B. K. Cho, A. Jain, S. M. Gruner and U. Wiesner, Science, 2004, 305, 1598-1601.

13 B. K. Cho, A. Jain, J. Nieberle, S. Mahajan, U. Wiesner, S. M. Gruner, S. Turk and H. J. Rader, Macromolecules, 2004, 37, $4227-4234$ 
14 G. Ungar, Y. S. Liu, X. B. Zeng, V. Percec and W. D. Cho, Science, 2003, 299, 1208-1211.

15 V. Percec, G. Johansson, G. Ungar and J. P. Zhou, J. Am. Chem. Soc., 1996, 118, 9855-9866.

16 M. Marcos, R. Martín-Rapún, A. Omenat, J. Barberá and J. L. Serrano, Chemistry of Materials, 2006, 18, 1206-1212.

17 J. M. Rueff, J. Barbera, B. Donnio, D. Guillon, M. Marcos and J. L. Serrano, Macromolecules, 2003, 36, 8368-8375.

18 M. Marcos, R. Martín-Rapún, A. Omenat and J. L. Serrano, Chem. Soc. Rev., 2007, 36, 1889-1901.

19 B. K. Cho, A. Jain, S. Mahajan, H. Ow, S. M. Gruner and U. Wiesner, J. Am. Chem. Soc., 2004, 126, 4070-4071.

20 M. Ornatska, S. Peleshanko, K. L. Genson, B. Rybak, K. N. Bergman and V. V. Tsukruk, J. Am. Chem. Soc., 2004, 126, 9675-9684.

21 Y. W. Chung, B. I. Lee, H. Y. Kim, U. Wiesner and B. K. Cho, Journal of Polymer Science Part a-Polymer Chemistry, 2007, 45, 4988-4994.

22 V. Percec, M. Glodde, T. K. Bera, Y. Miura, I. Shiyanovskaya, K. D. Singer, V. S. K. Balagurusamy, P. A. Heiney, I. Schnell, A. Rapp, H. W. Spiess, S. D. Hudson and H. Duan, Nature, 2002, 419, 384-387.
23 L. Gehringer, D. Guillon and B. Donnio, Macromolecules, 2003, 36, 5593-5601.

24 O. Spalla, in Neutrons, X-Rays and Light: Scattering Methods applied to Soft Condensed Matter, ed. T. Z. Peter Lindner, Elsevier, Amsterdam, 2002, pp. 49-71.

25 P. Fuchs, C. Tschierske, K. Raith, K. Das and S. Diele, Angew. Chem., Int. Ed., 2002, 41, 628-631.

26 X. H. Cheng, M. K. Das, S. Diele and C. Tschierske, Langmuir, 2002, 18, 6521-6529.

27 N. Canilho, E. Kasëmi, A. D. Schlüter, J. Ruokolainen and R. Mezzenga, Macromolecules, 2007, 40, 7609-7616.

28 N. Canilho, E. Kasëmi, A. D. Schlüter and R. Mezzenga, Macromolecules, 2007, 40, 2822-2830.

29 G. H. Fredrickson, The Equilibrium Theory of Inhomogeneous Polymers, Oxford University Press, 2006.

30 S. D. Hudson, H.-T. Jung, V. Percec, W.-D. Cho, G. Johansson, G. Ungar and V. S. K. Balagurusamy, Science, 1997, 278, 449-452.

31 T. Thurn-Albrecht, J. Schotter, C. A. Kastle, N. Emley, T. Shibauchi, L. Krusin-Elbaum, K. Guarini, C. T. Black, M. T. Tuominen and T. P. Russell, Science, 2000, 290, 2126-2129.

32 L. J. Shu, T. Schäfer, and A. D. Schlüter, Macromolecules, 2000, 33, $4321-4328$ 